\title{
Hemólise em circulação extracorpórea: estudo comparativo entre bomba de rolete e bomba centrífuga
}

\author{
Paulo M. PÊGO-FERNANDES*, Flávio MIURA*, Sérgio S. HIGA*, Luiz Felipe P. MOREIRA*, Luís Alberto
} DALLAN*, Dalton A. F. CHAMONE*, Sérgio de Almeida OLIVEIRA*, Noedir A. G. STOLF*, Adib D. JATENE*

PÊGO-FERNANDES, P. M.; MIURA, F.; HIGA, S. S.; MOREIRA, L. F. P.; DALLAN, L. A.; CHAMONE, D. A. F.; OLIVEIRA, S. A.; STOLF, N. A. G.; JATENE, A. D. - Hemólise em circulaçāo extracorpórea: estudo comparativo entre bomba de rolete e bomba centrífuga. Rev. Bras. Cir. Cardiovasc., 4(3): 220-224, 1989.

RESUMO: $O$ uso de bomba centrífuga como suporte circulatório para pacientes em choque cardiogênico, após a realização de cirurgia cardiaca e como suporte para pacientes com falência cardiaca que estão aguardando doação para transplante cardiaco, tem sido progressivamente ampliado. Alguns centros utilizam a bomba centrifuga em circulaçāo extracorpórea de rotina, como substituto do rolete arterial,. No INCOR, operamos dois grupos de pacientes triarteriais submetidos a revascularização do miocárdio, operados pelo mesmo cirurgião, com o mesmo método de proteção miocárdica (cardioplegia cristalóide, hipotermia sistêmica a $28^{\circ} \mathrm{C}$ e tópica com soro fisiológico). Todos os parâmetros dos dois grupos foram sem diferença estatística no pré-operatório: idade, sexo, superfície corpórea e parâmetros hematológicos. Foram operados 27 pacientes consecutivos e divididos, alternadamente, em 13 pacientes com bomba centrifuga e 14 com rolete arterial. $\mathrm{O}$ oxigenador utilizado em todos foi o de bolhas da Macchi. O perfusionista foi sempre o mesmo. $\mathrm{O}$ tempo de perfusão médio foi de 105 minutos no Grupo 1 (rolete) e 103 minutos no Grupo 2 (bomba centrífuga). Analisamos os seguintes parâmetros: haptoglobina (HP), tempo de tromboplastina parcial ativada (TTPA), tempo de trombina (TT) e número e plaquetas pré e pós circulação extracorpórea e, comparando-se os dois grupos, não houve diferença estatística significante entre eles, nos diversos parâmetros. Concluímos que, para circulação extracorpórea com duração habitual, nāo há diferença hematológica no uso da bomba centrifuga em relação ao rolete arterial.

DESCRITORES: hemólise em circulação extracorpórea; circulação extracorpórea, bomba de rolete; circulação extracorpórea, bomba centrífuga.

\section{INTRODUÇĀO}

O uso de bomba centrifuga como suporte circulatório para pacientes em choque cardiogênico, após a realização de cirurgia cardíaca e como suporte para pacientes com falência cardíaca que estão aguardando doação para transplante cardiaco, tem sido progressivamente ampliado ${ }^{5}$. Alguns autores utilizam a bomba centrífuga em circulação extracorpórea (CEC) de rotina como substituto do rolete arterial. Significativas alteraçōes na hemostasia e no grau de hemólise são produzidos pelo uso de circulação extracorpórea (CEC). Quando a CEC é utilizada em cirurgias cardíacas de longa duração ou com suporte respiratório, o tempo pode durar várias horas ou alguns dias ${ }^{7}$.

\footnotetext{
Trabalho realizado no Instituto do Coraçăo do Hospital das Clínicas da Faculdade de Medicina da Universidade de São Paulo. Sâo Paulo, SP. Brasil.

Apresentado ao 16: Congresso Nacional de Cirurgia Cardiaca. São Paulo, SP, 7 e 8 de abril, 1989.

- Do Instituto do Coraçáo do Hospital das Clinicas da Faculdade de Medicina da Universidade de Sâo Paulo.

Endereço para separatas: Paulo Pêgo-Fernandes. Av. Dr. Enéas de Carvalho Aguiar, 44. Divisão Cirúrgica. 05403 Săo Paulo, SP, Brasil.
} 
PÊGO-FERNANDES, P. M.; MIURA, F.; HIGA, S. S.; MOREIRA, L. F. P.; DALLAN, L. A.; CHAMONE, D. A. F.; OLIVEIRA, S. A.; STOLF, N. A. G.; JATENE, A. D. - Hemólise em circulação extracorpórea: estudo comparativo entre bomba de rolete e bomba centrifuga. Rev. Bras. Cir. Cardiovasc., 4(3): 220-224, 1989.

O baixo grau de hemólise causado pela bomba centrífuga é uma indicação para procedimentos mais longos. Com o objetivo de analisar se o grau de alteraçōes na hemostasia e o grau de hemólise em circulação extracorpórea de rotina são significantemente diferentes daquele causado pela bomba de rolete, realizamos este estudo.

\section{CASUÍSTICA E MÉTODOS}

Foram estudados, no Instituto do Coração (INCOR), dois grupos de pacientes triarteriais submetidos a revascularização do miocárdio. Esses doentes foram operados pelo mesmo cirurgião, com o mesmo perfusionista e com o mesmo método de proteção miocárdica (cardioplegia cristalóide, hipotermia sistêmica a $28^{\circ} \mathrm{C}$ e tópica com soro fisiológico à $4^{\circ} \mathrm{C}$ ). Foram 20 homens e sete mulheres sendo que a idade variou de 33 a 77 anos, com média de 58,7 anos. O peso variou de $40,0 \mathrm{~kg}$ a $85,5 \mathrm{~kg}$ com média de $66,8 \mathrm{~kg}$ e a superfície corpórea variou de $1,3 \mathrm{~m}^{2}$ a $2,0 \mathrm{~m}^{2}$, com média de $1,7 \mathrm{~m}^{2}$. Analisamos os seguintes parâmetros hematológicos: haptoglobina (HP), tempo de tromboplastina parcial ativada (TTPA), tempo de trombina (TT), hemoglobina livre (Hb livre) e número de plaquetas no pré e pós CEC. Em relação aos parâmetros hematológicos pré-CEC, o TP variou de $13,6 \mathrm{~s}$ a $19,8 \mathrm{~s}$, com média de $16,6 \mathrm{~s}$; o TTPA variou de $41,5 \mathrm{~s}$ a $67,5 \mathrm{~s}$, com média de 54,3 $\mathrm{s} ;$ o TT variou de $10,7 \mathrm{~s}$ a $28,3 \mathrm{~s}$, com média de 14,4 $\mathrm{s}$; a contagem de plaquetas variou de $69.000 / \mathrm{mm}^{3}$ a $488.000 / \mathrm{mm}^{3}$, com média de $179.961 / \mathrm{mm}^{3}$, a haptoglobina variou de 0,35 a $3,5 \mathrm{~g} / \mathrm{l}$, com média de $1,8 \mathrm{~g} / \mathrm{l}$; e a hemoglobina livre variou de 0,7 a $23 \mathrm{mg} / \mathrm{dl}$, com média de $6,4 \mathrm{mg} / \mathrm{dl}$.

Foram estatisticamente semelhantes no pré-operatório todos os parâmetros biométricos e hematológicos dos dois grupos. Os 27 pacientes foram consecutivamente operados, divididos alternadamente em 13 pacientes com bomba centrífuga e 14 com rolete arterial. $O$ oxigenador utilizado em todos foi o de bolhas da Macchi, a bomba centrifuga foi a Biomedicus e a bomba de roletes foi a da Bioengenharia INCOR. O tempo médio de perfusāo foi de 105 minutos no Grupo 1 (rolete) e 103 minutos no Grupo 2 (bomba centrífuga).

O método estatístico utilizado foi o do Qui-quadrado, considerando-se dados significativos $P<0,05$.

\section{RESULTADOS}

$\mathrm{Na}$ análise dos parâmetros pós-CEC, o TP variou de $16,8 \mathrm{~s}$ a $22,4 \mathrm{~s}$, com média de $19,3 \mathrm{~s}$ com bomba centrífuga e variou de $14,2 \mathrm{~s}$ a $30,5 \mathrm{~s}$, com média de 17,0 s com bomba de rolete ( $p=0,2264$ ) (Gráfico 1).

O TTPA variou de $42,4 \mathrm{~s}$ a $58,3 \mathrm{~s}$, com média de 49,9 s com bomba centrífuga e variou de 45,0 s a 59,9
GRÁFICO 1

MÉDIAS DOS COAGULOGRAMAS APOS CEC EM BOMBA CENTRIFUGA E DE ROLETE.

MÉdia dos valores do COAgULOGRAMA

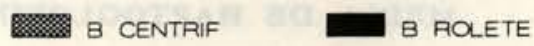

60

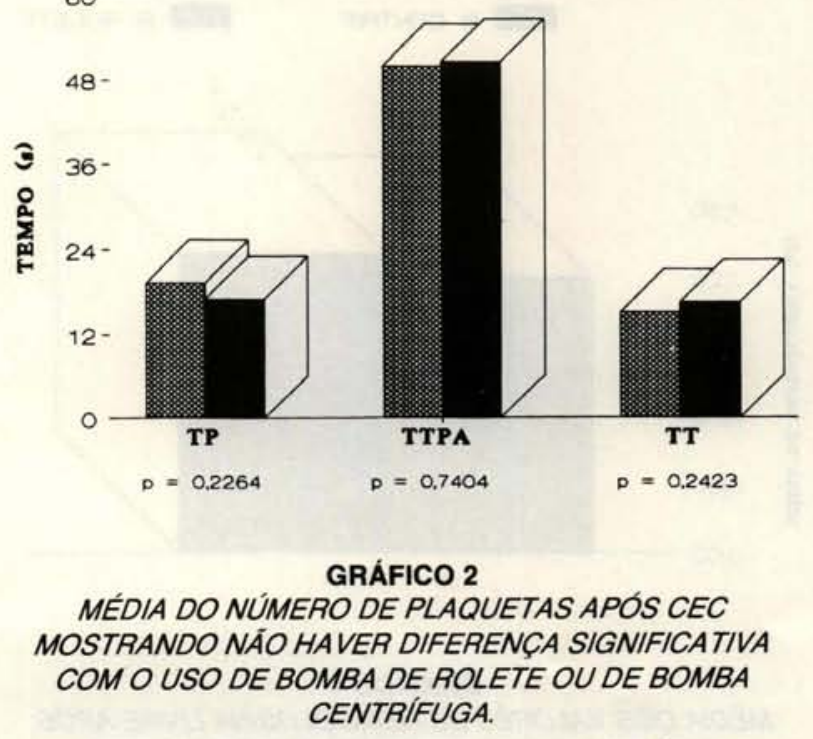

MÉdia de plaquetas pós-cec

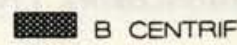

B ROLETE

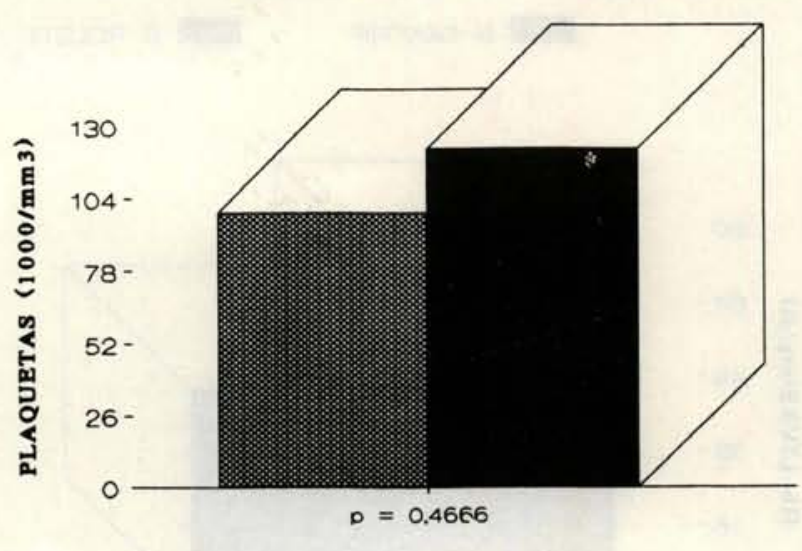

$\mathrm{s}$, com média de $50,6 \mathrm{~s}$ com bomba de rolete $(\mathrm{p}=$ 0,7404) (Gráfico 1).

O TT variou de $12,6 \mathrm{~s}$ a $18,0 \mathrm{~s}$, com média de 15,1 s com bomba centrífuga e variou de 13,3 s a 25,7 $\mathrm{s}$, com média de $16,5 \mathrm{~s}$ com bomba de rolete $(\mathrm{p}=$ 0,2423) (Gráfico 1).

A contagem de plaquetas variou de $64.000 / \mathrm{mm}^{3}$ a $130.000 / \mathrm{mm}^{3} \mathrm{com}$ média de $99.000 / \mathrm{mm}^{3} \mathrm{com}$ bomba centrifuga e variou de $53.000 / \mathrm{mm}^{3}$ a $157.000 / \mathrm{mm}^{3} \mathrm{com}$ média de $121.846 / \mathrm{mm}^{3} \mathrm{com}$ bomba de rolete $(\mathrm{p}=$ 0,4666) (Gráfico 2). 
PÊGO-FERNANDES, P. M.; MIURA, F.; HIGA, S. S.; MOREIRA, L. F. P.; DALLAN, L. A.; CHAMONE, D. A. F.; OLIVEIRA, S. A.; STOLF, N. A. G.; JATENE, A. D. - Hemólise em circulaçāo extracorpórea: estudo comparativo entre bomba de rolete e bomba centrifuga. Rev. Bras. Cir. Cardiovasc., 4(3): 220-224, 1989.

GRÁFICO 3

MÉDIA DOS VALORES DE HAPTOGLOBINA APÓS CEC, SEM DIFERENCCA SIGNIFICATIVA ENTRE AS DUAS BOMBAS.

MÉdia DE HAPTOGLOBINA PÓS-CEC B CENTRIF B ROLETE

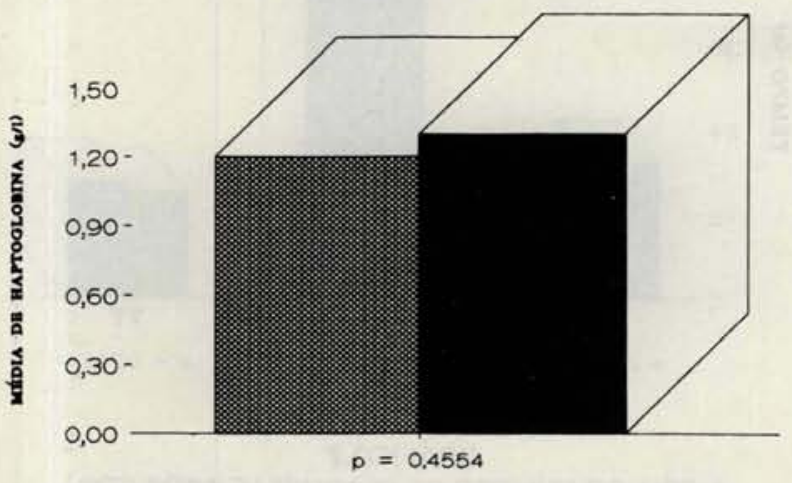

GRÁFICO 4

MÉDIA DOS VALORES DE HEMOGLOBINA LIVRE APÓS CEC COM BOMBA CENTRIFIGA E DE ROLETE.

MÉDIA DE HEMOGLOBINA LIVRE PÓS-CEC

B CENTRIF B ROLETE

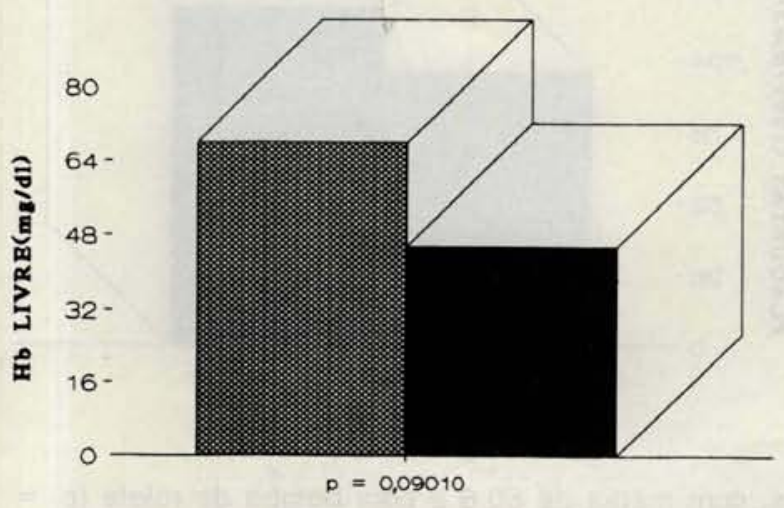

A haptoglobina variou de 0,25 a 2,3 g/l, com média de $1,2 \mathrm{~g} / \mathrm{l}$ com bomba centrífuga e variou de 0,45 a $2,3 \mathrm{~g} / \mathrm{l}$, com média de $1,3 \mathrm{~g} / \mathrm{com}$ bomba de rolete ( $\mathrm{p}$ = 0,4954) (Gráfico 3).

A hemoglobina livre variou de 12,2 a $183,5 \mathrm{mg} / \mathrm{dl}$, com média de $67,9 \mathrm{mg} / \mathrm{dl}$ com bomba centrifuga e variou de 29,0 a $81,2 \mathrm{mg} / \mathrm{dl}$, com média de $45,5 \mathrm{mg} / \mathrm{dl}$ com bomba de rolete ( $p=0,0910$ ) (Gráfico 4$)$.
Como podemos observar, a análise estatística do Qui-quadrado demonstrou nảo haver diferenças hematológicas significativas entre esses dois sistemas.

\section{DISCUSSĀO}

A bomba centrifuga Biomedicus consiste de um cone de acrílico dentro do qual existem três cones magnéticos acoplados e rotatórios que movem o sangue através de força centrífuga (Figura 1). Esse desenho possibilita várias vantagens sobre outros sistemas, pois tem como princípio um vórtex que permite o fluxo unidirecional sem o uso de válvulas e permite o deslizamento suave e contínuo através do dispositivo, de modo que complicaçōes trombóticas e embólicas sāo minimizadas ${ }^{6}$ e a menor turbulência gerada reduz os danos aos elementos figurados do sangue ${ }^{1}$. A bomba centrifuga tem menor risco de formar pressão negativa (inclusive pressão negativa de cavitação) ${ }^{4,9}$ e não tem 0 risco de rotura da linha arterial por aumento de pressão devido à oclusão súbita. As desvantagens incluem o uso de um sistema mais sofisticado e um custo maior.

MANDL ${ }^{10}$, em 1977, comparou o uso de bomba centrifuga e bomba de rolete, e achou que a incidência de embolia foi significativamente menor com a bomba centrifuga. Ele explicou esse fato mostrando que as altas

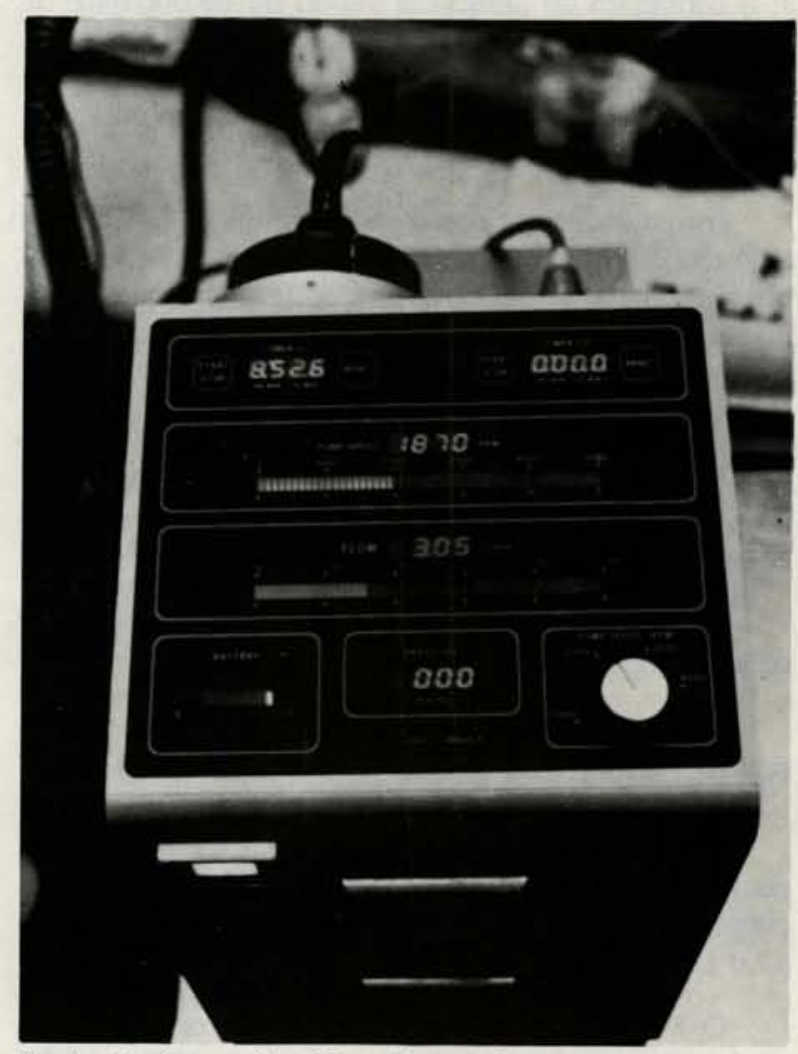

Fig. 1 - Bomba centrifuga Biomedicus em funcionamento. 
PÊGO-FERNANDES, P. M.; MIURA, F.; HIGA, S. S.; MOREIRA, L. F. P.; DALLAN, L. A.; CHAMONE, D. A. F.; OLIVEIRA, S. A.; STOLF, N. A. G.; JATENE, A. D. - Hemólise em circulação extracorpórea: estudo comparativo entre bomba de rolete e bomba centrifuga. Rev. Bras. Cir. Cardiovasc., 4(3): 220-224, 1989.

taxas de pressāo e a pressão negativa transitória produzida pelo rolete podem levar à formação de êmbolos. Nos nossos casos, não houve manifestação clínica de embolias.

Alguns autores ${ }^{7,11}$ demonstraram, clínica e experimentalmente, a superioridade hematológica e a menor incidência de êmbolos da bomba centrífuga em relação à bomba de roletes. Em estudo clínico, WHEELDON et alii ${ }^{11}$, estudando, retrospectivamente, 16 pacientes (divididos em dois grupos de oito para cada tipo de bomba) que foram submetidos a cirurgia de revascularização do miocárdio (com tempo de perfusão de 94 minutos com bomba centrífuga e 105 minutos com bomba de rolete), demonstraram maior preservação do número de plaquetas, menor atividade do complemento e menor índice de miocroêmbolos ( $800 \%$ menor) da bomba centrífuga em relação à bomba de roletes Stockert. Esses autores não notaram, porém, diferença na evolução clíni$\mathrm{ca}$, nesse pequeno número de doentes.

Investigadores demonstraram redução dos níveis séricos de hemoglobina livre em experimentos longos, utilizando cães e carneiros quando a bomba de rolete era substituída pela bomba centrífuga, concluindo que a bomba de rolete possa não ser a melhor bomba para suporte cardíaco de longa duração ${ }^{2 \cdot 4,8}$.

Em relação ao nosso estudo, não notamos diferenças clínicas ou hematológicas entre os dois sistemas. Acreditamos que, por esses pacientes terem sido submetidos à CEC por um tempo relativamente curto (média de 103 minutos), as diferenças entre os dois sistemas não se manifestaram. Baseados nos nossos dados, concluímos que, para CEC com duração habitual, em pacientes não obesos, eletivos e em boas condiçōes clínicas como os de nosso estudo, não há diferenças hematológicas em relação à bomba de rolete.

RBCCV 44205-96

PÊGO-FERNANDES, P. M.; MIURA, F.; HIGA, S. S.; MOREIRA, L. F. P.; DALLAN, L. A.; CHAMONE, D. A. F.; OLIVEIRA, S. A.; STOLF, N. A. G.; JATENE, A. D. - Hemolysis in extracorporeal circulation: a comparative study between roller and centrifugal pumps. Rev. Bras. Cir. Cardiovasc., 4(3):220-224, 1989.

ABSTRACT:The utilization of centrifugal pumps as circulatory support in patients with cardiogenic shock after cardiac surgery and as support in patients waiting for cardiac transplant has been progressively extended. Some centers utilize the centrifugal pump in routine extracorporeal circulation as a substitute for roller pump. At INCOR, we operared upon 2 groups of triple vessel disease patients submitted to coronary arterial bypass graft (CABG). They were operated upon by the same surgeon, with the same perfusionist and with the same perfusionist and the same myocardial protection method (cristaloid cardioplegia, systemic hipotermia at $28^{\circ} \mathrm{C}$ and topic hipotermia with $\mathrm{NaCl} 0,9 \%$ ). All pre-operatory parameters in both groups were statistically similar: age, sex, corporeal surface and analized hematologic parameters). Twenty-seven patients were consecutively and alternatively operated, 13 with centrifugal pump and 14 with roller pump. We utilized the Macchi bubble oxygenator. The average perfusion time was 105 minutes in the roller pump group and 103 minutes in the centrifugal pump group. We analized protrombin time, haptoglobin, activated partial tromboplastin time, trombin time and platelet number in the pre and pos extracorporeal circulation time, comparing both groups.

There was no statistical significant difference between the several parameters. We conclude that in habitual duration extracorporeal circulation there is no hematological difference between centrifugal and roller pumps.

DESCRIPTORS: hemolysis in extracorporeal circulation; extracorporeal circulation, roller pump; extacorporeal circulation, centrifugal pump.

\section{REFERÊNCIAS BIBLIOGRÁFICAS}

1 BLACKSHEAR, P. - Effects of the flow and turbulence on the formed elements of blood. In: Prostect heart valves, 1969. cap. 5 , p. 52-67.

2 CRANE, K. A.;BROWN, D.; ANDERSON, R.; BAGELMAN, K. M. - Further decrease in subclinical hemolysis utilizing $12,7 \mathrm{~mm}$ tubing in the arterial roller head. Ann. Thorac. Surg., 35: 463-465, 1983.
3 DIXON, C. M.; KAO, R. L.; MAGOVERN, G. J. - Left-ventricular assist with the new bio-pump $80 . \quad J$. ExtraCorp. Tech., 18: 117-121, 1986.

4 DIXON, C. M. \& MAGOVERN, G. J. - Evaluation of the Bio pump for long-term cardiac support without heparinization. J. Extra-Corporeal Tech., 14: 331-336, 1982. 
PÊGO-FERnANDES, P. M.; MIURA, F.; HIGA, S. S.; MOREIRA, L. F. P.; DALLAN, L. A.; CHAMONE, D. A. F.; OLIVEIRA, S. A.; STOLF, N. A. G.; JATENE, A. D. - Hemólise em circulação extracorpórea: estudo comparativo entre bomba de rolete e bomba centrifuga. Rev. Bras. Cir. Cardiovasc., 4(3): 220-224, 1989.

5 FERNANDES, P. M. P.; MOREIRA L. F. P.; JATENE, F. B.; AULER Jr., O. C.; MORAES, A. V.; JATENE, A. D. - Circulação assistida com bomba centrifuga de choque cardiogênico após cirurgia com extracorpórea. Arq. Bras. Cardiol., 56: 313-316, 1991.

6 FRAZIER, O. H. \& RADOVANCEVIC, B. - Ventricular assist devices. Card. Surg., 4: 335-347, 1990.

7 HARRY, R.; HOERR, J. R.; MICHAEL, F.; KRAEMER, J.; WILLIAMS, L.; SHERMAN, M. L.; RILEY, J. B.; CROWLEY, J. C.; SORENSEN, S. W. - In vitro comparison of the blood handling by the constrained vortex and twin roller pumps. J. Extra-Corp. Tech., 19: 316-321, 1987.
8 HERMANN, W.; STENZL, W.; TSCHELIESSNIGG, K. H.; DACAR, D.; SEMMELROCK, H. J. - Die Zentrifugalblutpumpe im Experiment. Zentralbl. Chir., 106: 243-1255, 1981.

9 LYNCH, M. F.; PETERSON, D.; BAKER, V. - Centrifugal blood pumping for open heart surgery. Minnesota Med., 25: 536-537, 1978.

10 MANDL, J. P. - Comparison of emboli production between a constrained vortex pump and a roller pump. Am. Sect. Proc., 27, 1977.

11 WHEELDON, D. R.; BETHUNE, B. W.; GILL, R. D. Vortex pumping for routine cardiac surgery: a comparative study. Perfusion, 5: 135-143, 1990. 